\title{
Development of an Intact Mammalian System for High-resolution Imaging by Cryo- Electron Tomography
}

\author{
Xia Li1 ${ }^{1,2}$, Donghyun Park ${ }^{1}$, Yunjie Chang ${ }^{1}$, Abhijith Radhakrishnan ${ }^{3}$, Hangjun $\mathrm{Wu}^{4}$, Jun Liu ${ }^{*}$ \\ 1. Department of Microbial Pathogenesis and Microbial Science Institute, Yale University School of \\ Medicine, New Haven, CT, USA \\ 2. Institute of Special Environmental Medicine and Co-innovation Center of Neuroregeneration, Nantong \\ University, Nantong, Jiangsu, China \\ 3. Department of Cell Biology, Yale University School of Medicine, New Haven, CT, USA \\ 4. Center of Cryo Electron Microscopy and Department of Pathology of Sir Run Run Shaw Hospital, \\ Zhejiang University School of Medicine, Hangzhou, China \\ * Corresponding author: jliu@yale.edu
}

Cryo-electron tomography (cryo-ET) has been extensively used to characterize thin specimens such as prokaryotes and viruses. However, imaging eukaryotic cells remains challenging due to their thicker profile $(>500 \mathrm{~nm})$. A few selective areas such as the thin periphery of endothelial cells, epithelial cells, and primary neuronal synapses are accessible to cryo-ET imaging. To overcome this limitation, a number of approaches, including cryo-electron microscopy of vitreous sections (CEMOVIS) and cryo-Focused Ion Beam (FIB) milling, have been utilized to generate thin sections (or lamella) of vitrified eukaryotic specimens. Nevertheless, it remains challenging to reproducibly obtain high-quality vitreous sections (or lamella) by using CEMOVIS and FIB milling.

We developed a mammalian model system based on nerve growth factor (NGF) differentiated rat adrenal pheochromocytoma (PC12) cells - a cell line, originated from a tumor of adrenal medulla chromaffin cells, that is widely used in studying neuro-secretion, cell signaling, proliferation, and differentiation. After treatment with NGF, PC12 cells differentiate into neuron-like cells. The neurites protruding from the cellular soma (cell body) are often less than $500 \mathrm{~nm}$ in thickness. PC12 cells are easier to culture and manipulate than primary neuronal cultures. Importantly, the unique properties of NGF differentiated PC12 cells facilitate multiscale tracking by Correlative Light and Electron Microscopy (CLEM)and highresolution imaging by cryo-ET. Our general workflow consists of: 1) preparing neurite varicosities on EM grids, 2) fluorescent light microscopy (fLM) imaging of neurites in room-temperature and/or cryogenic condition to define specific targets, and 3) coordinating the fLM targets in a transmission electron microscope for high resolution cryo-ET imaging.

Preparation of neurite varicosities on EM grids. Quantifoil R2/1 Au-grids were glow discharged for $25 \mathrm{sec}$ and then sterilized in ethanol under UV-light and coated with human plasma fibronectin. PC12 cells were all maintained at $37^{\circ} \mathrm{C}$ in a $95 \%$ humidified incubator with $5 \% \mathrm{CO}_{2} .24$ hours before seeding cells onto EM grids, the media was replaced with complete media supplemented with $100 \mathrm{ng} / \mathrm{ml}$ NGF to differentiate the cells. Grids were blotted from the back side with filter paper and plunge-frozen into liquid ethane. After NGF differentiation for 3-7 days, multiple neurites ( $>100 \mu \mathrm{m}$ in length) extend from the soma with circular varicosities throughout the entire length. These thinned varicosities are ideally suited for cryo-ET imaging of various cellular features with high contrast and high resolution (Figure). To identify area of interests, cryopreserved grids can also be imaged with a cryo-CLEM system. Fluorescent signals can be used to select as positions of interest (POIs). The coordinates data of POIs are then transferred onto a microscope computer. 
Cryo-ET data acquisition and reconstruction. SerialEM was used to locate the specific regions and collect low-dose, single-axis tilt series with dose fraction mode and Volta Phase Plate (VPP). Images in tilt series were first aligned using MotionCor2 [1]. Subsequently, tilt series was aligned using the IMOD [2], with fiducial markers or fiducial-free cross correlation, and reconstructed by using IMOD. EMAN2.2[3] were used to generate 3D representations of cellular features.

In summary, we have demonstrated that the NGF differentiated PC12 cell is an accessible mammalian cell system for high-resolution cryo-ET imaging. We have developed a novel imaging workflow that effectively combines the advanced improvements in CLEM and cryo-ET to routinely produce threedimensional and molecular resolution snapshots of neurite varicosities containing intact organelles and molecular machines in their native and functional environments.

\section{References:}

[1] Zheng, S.Q., et al., Nat Methods 14(4) (2017), p. 331.

[2] Mastronarde, D.N. and S.R. Held, J Struct Biol. 197(2) (2017), p. 102.

[3] Chen, M., et al., Nat Methods 14(10) (2017), p. 983.
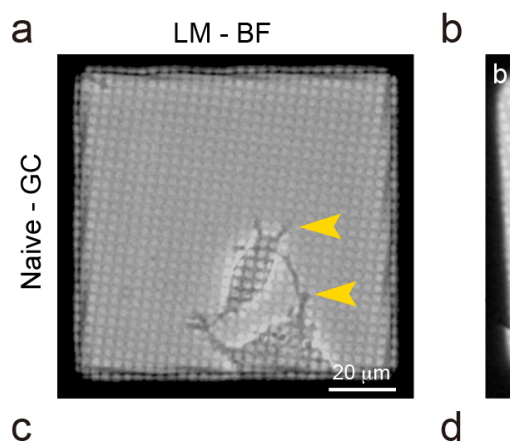

EM - 220x
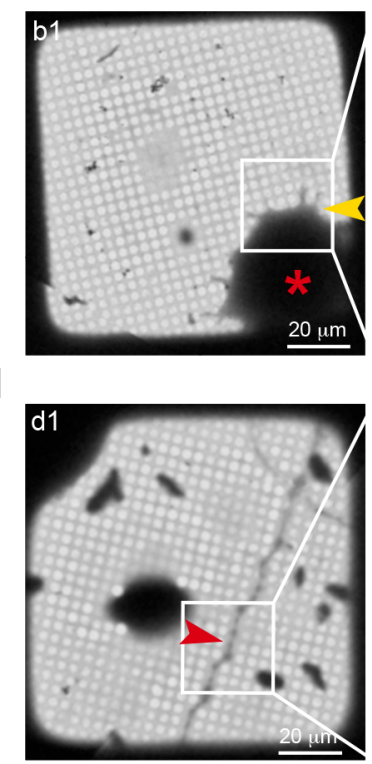

EM - 3600x
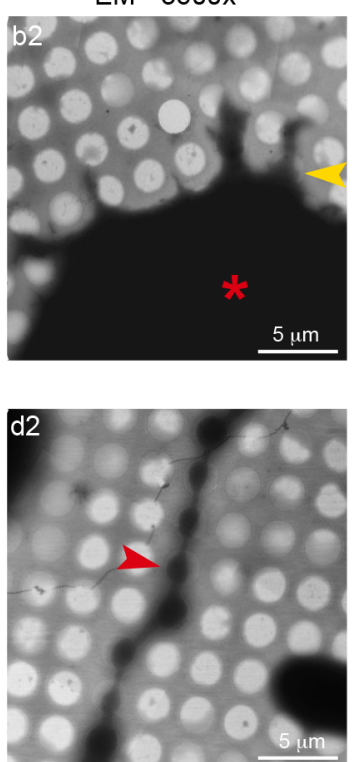

Reconstruction
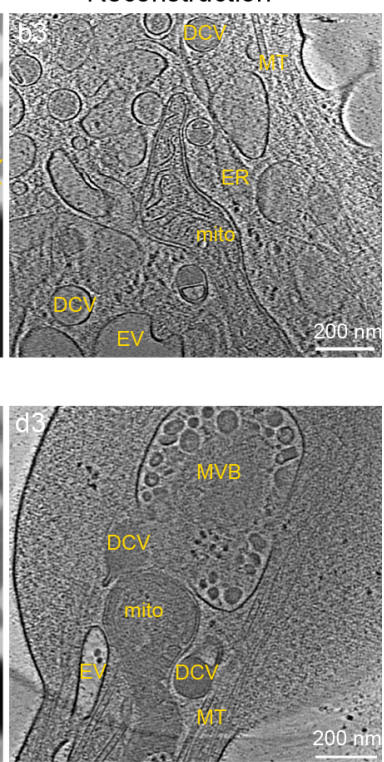

Figure 1. PC12 cells differentiated with NGF increases areas optimal for cryo-ET imaging.

(a-b) Naïve PC12 cells on EM grids. Bright field (BF) image shows naïve PC12 cells extending short growth cone protrusions. (b1) 220× magnification montage of cryogenic naïve PC12 cells showing thick soma and thin growth cones. (b2) 3600× magnification montage of boxed area in panel b1. (b3) Tomographic slice showing a representative protrusion. Dense core vesicles (DCV), endoplasmic reticulum (ER), endosome vesicles (EV), mitochondria (Mito), and microtubules (MT) are annotated. (cd) Varicosity of PC12 cells differentiated with NGF for 7 days. BF images showing NGF differentiation triggered PC12 cells to yield long processes shown in panel c and e. (d1) 220× magnification montage of differentiated neurites with varicosities along the neurite. (d2) 3600× magnification montage of boxed area in panel d1. (d3) A tomographic slice showing representative varicose. DCV, Mito, MT, EV, multivesicular bodies (MVB), actin bundles, and ribosomes are annotated. 